\title{
The CBI Receptor as an Important Mediator of Hedonic Reward Processing
}

\author{
Chris M Friemel', Andreas Zimmer² and Miriam Schneider*,' \\ 'Reseach Group Developmental Neuropsychopharmacology, Institute of Psychopharmacology, Central Institute of Mental Health, Medical \\ Faculty Mannheim, Heidelberg University, Mannheim, Germany; ${ }^{2}$ Institute of Molecular Psychiatry, University of Bonn, Bonn, Germany
}

\begin{abstract}
The endocannabinoid (ECB) system has emerged recently as a key mediator for reward processing. It is well known that cannabinoids affect appetitive learning processes and can induce reinforcing and rewarding effects. However, the involvement of the ECB system in hedonic aspects of reward-related behavior is not completely understood. With the present study, we investigated the modulatory role of the ECB system on hedonic perception, measured by the pleasure attenuated startle (PAS) paradigm for a palatable food reward. Here, a conditioned odor is thought to induce a pleasant affective state that attenuates an aversive reflex - the acoustic startle response. Modulatory effects of the CBI receptor antagonist/inverse agonist SR I 4I I7I 6 and the cannabinoid agonist WIN 55 2I2-2 on PAS were examined in rats. PAS was also measured in $\mathrm{CBI}$ receptor knockout $(\mathrm{KO})$ and wild-type (WT) mice. Pharmacological inhibition as well as the absence of CBI receptors was found to reduce PAS, whereas WIN 55212 -2 administration increased PAS. Finally, presentation of a conditioned reward cue was found to induce striatal FosB/ $\mathrm{F}$ osB expression in WT mice, but not in $\mathrm{KO}$ mice, indicating a reduced stimulation of reward-related brain regions in conditioned $\mathrm{KO}$ mice by odor presentation. We here show that in addition to our previous studies in rats, PAS may also serve as a valuable and suitable measure to assess hedonic processing in mice. Our data further indicate that the ECB system, and in particular CBI receptor signaling, appears to be highly important for the mediation of hedonic aspects of reward processing.

Neuropsychopharmacology (2014) 39, 2387-2396; doi:I0.I038/npp.2014.86; published online I4 May 20I4
\end{abstract}

\section{INTRODUCTION}

From an evolutionary perspective, it is of utmost importance to reinforce actions that are crucial for survival and therefore to support and encourage vital processes, such as eating, social contact, and reproduction (Schultz, 2010). Events, behavioral actions, or objects that satisfy these basic needs are therefore generally considered as primary rewards. These processes are so elementary for survival that it is not surprising for a phylogenetically ancient system, such as the endocannabinoid (ECB) system (Elphick, 2012), to be strongly involved in the neurobiological mechanisms mediating reward perception and processing. The term 'reward' is complex and includes a variety of different connotations that are mainly linked to the hedonic value, reward motivation, learning and extinction processes, and anticipation or expectation for rewarding stimuli (Salamone $e t a l$, 2007; Barbano and Cador, 2007; Schneider et al, 2010). Dysfunctions of reward processing and altered reward

*Correspondence: Dr M Schneider, Reseach Group Developmental Neuropsychopharmacology, Institute of Psychopharmacology, Central Institute of Mental Health, Medical Faculty Mannheim, University of Heidelberg, J5, 68I59 Mannheim, Germany, Tel: + I 49621 I7036269, Fax: + 4962 I 17036255, E-mail: miriam.schneider@zi-mannheim.de Received 25 November 2013; revised 4 April 20I4; accepted 6 April 2014; accepted article preview online 10 April 2014 perception are linked to various neuropsychiatric disorders (eg, addiction, depression, and schizophrenia). This is particularly true for the experience of pleasure, which is essential for a normal sense of well-being (Berridge and Kringelbach, 2008). A detailed knowledge of neurocircuits and mechanisms involved in different aspects of reward processing is crucial to increase our understanding on the potential etiology and neuropathology of anhedonic states and behavior.

Components of the ECB system are widely distributed throughout the brain reward circuits (Herkenham et al, 1990; Egertova et al, 2003; Solinas et al, 2008), and ЕСВ signaling exerts an important modulatory influence on all other neurotransmitter systems involved in the mediation of reward-related behaviors (eg, dopamine, glutamate, and endogenous opioids). A clear involvement of ECB signaling in learning processes, neuroplasticity, motivational behavior (eg, operant conditioning), and reward 'wanting' has been reported (Fattore et al, 2010; Ameri, 1999; Solinas et al, 2008). However, the role of the ECB system in processes of hedonic experiences is only poorly understood. It is believed that there are three main reward components ('liking', 'wanting', and learning) and pleasure or hedonic 'liking' has probably been the least addressed in neuroscience studies (Berridge and Kringelbach, 2008). This is mainly due to the lack of appropriate behavioral paradigms for assessing pleasure and the hedonic value of a reward in 
laboratory animals. Nevertheless, a prominent feature of Cannabis intake reported from human users is an initial period of euphoria and relaxation (Ameri, 1999). It has therefore been suggested that the ECB system and cannabinoids might act in the brain to increase the hedonic impact of a reward (Mahler et al, 2007; Kirkham, 2009; Jarrett et al, 2007; Cota et al, 2006; Solinas et al, 2008), most probably by a close interaction with the endogenous opioid system (Cota et al, 2006). The involvement of the ECB system in pleasure and hedonic reward aspects was mainly assessed by measuring the intake of palatable food (reviewed by Kirkham, 2009) and also through taste reactivity studies (Jarrett et al, 2007; Mahler et al, 2007), where an inhibition of CB1 receptors was found to decrease consumption and oral 'liking' responses. However, it is still not understood whether these findings may relate to the effects of cannabinoids on orosensory taste perception or if the ECB system is indeed involved in the 'pleasure' of eating.

We have recently established a novel protocol for assessing appetitive modulations of the acoustic startle reflex (ASR) as a valid psychophysiological measure of pleasant emotional states in rats (Schneider and Spanagel, 2008; Schneider et al, 2010; Brand et al, 2012; Enkel et al, 2010). Here, a neutral olfactory stimulus is associated in an appetitive conditioning procedure with a reward and subsequently reduces the ASR. The benefits and the usefulness of the ASR in the context of emotional modulations were first reported in humans (Vrana et al, 1988), indicating the startle probe procedure as a very effective means for assessing emotions. In particular, the fear-potentiated startle paradigm, the augmentation of the ASR in an aversive emotional state, has been used since then to investigate the pharmacological and neuronal basics of fear in both humans and rats (eg, Davis, 1998; Lang, 1995; Davis et al, 2003; Norrholm et al, 2006). However, it was found in humans that the amplitude of the ASR is not only increased during states of fear and anxiety but also decreases if elicited in a pleasant emotional context (Vrana et al, 1988; Lang et al, 1990). This latter phenomenon can also be assessed in rats and was termed PAS (pleasure-attenuated startle) (Schmid et al, 1995). As PAS can be measured in humans as well as in rats, it serves as a cross-species model to measure reward-related affect (Koch et al, 2000). It has been suggested before that the impact of emotions on ASR modulation is mediated by emotional priming, in which emotions are viewed as action dispositions that prepare an organism to respond to environmental stimuli, ultimately improving survival by inducing approach or avoidance responses (Lang, 1995). PAS has been suggested to result from negative motivational priming, whereby hedonic emotions suppress or 'de-energize' inappropriate behaviors (like the defensive startle response) in a pleasant emotional state. According to this concept, the positive motivation of reward expectancy interacts with the brain sites that mediate aversive responses and thereby suppresses the ASR (Lang 1995; Koch et al, 2000). PAS thereby enables the direct assessment of hedonic qualities of a reward in rodent models, independent of acute sensory food reward perception and without operant requirements, and it appears not to be related to attentional alterations or a more general arousal elicited by odor presentation (Schneider and Spanagel, 2008). The PAS paradigm offers a completely new approach for investigating the neural mechanisms of appetitive and hedonic emotions, because it measures the physiological reduction of a reflex, rather than reinforcing instrumental or active behavioral responding (as required for conditioned place preference, operant self-administration, or intake procedures) (Lang, 1995; Koch et al, 2000; Schmid et al, 1995; Schneider and Spanagel, 2008).

For the present study, we used our established PAS paradigm (with the olfactory conditioning procedure) to clarify the modulatory impact of CB1 receptor signaling in hedonic reward processing. As PAS has so far only been assessed in rats, another aim of this study was to establish and validate the paradigm in laboratory mice. The exposure to natural incentives and drugs of abuse leads to neuroplastic changes in reward-related brain regions and we could show recently that acute presentation of an appetitively conditioned olfactory stimulus in rats stimulates the immediate early gene $c$-Fos in striatal regions (Friemel et al, 2010). For the present study, we aimed to expand our findings to explore the involvement of other transcription factors. To investigate potential differences in neuronal reward processing in WT and KO mice, we additionally examined the ability of an conditioned odor to stimulate striatal FosB/ $\Delta$ FosB expression.

\section{SUBJECTS AND METHODS}

\section{Subjects}

A total 132 of animals were used for the present study. Male adult Wistar rats were purchased from Wistar Harlan Laboratories (AN Venray, The Netherlands). Male adult wild-type (WT) and CB1 receptor knockout (CB1 KO) mice with C57BL/6J genetic background bred at the University of Bonn (Zimmer et al, 1999) were transported to Mannheim (Germany). Animals were allowed to recover from transportation and were habituated to the new environment for at least 7 days after arrival. All animals used in the present experiments were matched for age (rats: 4 months; mice: 2-3 months). Mice were housed individually to avoid whisker barbering and aggressive behavior in Makrolon cages (Eurostandard type II) and rats were housed in groups of four (Eurostandard type IV) in an animal colony room on a $12 \mathrm{~h}$ light-dark schedule (lights on 0700-1900 hours). Animals received free access to water and standard lab food during the first week after arrival and were than maintained on approximately $95-97 \%$ of their free-feeding bodyweight during behavioral testing when the high caloric sweetened condensed milk was provided.

All experiments were carried out in accordance with the Guide for the Care and Use of Laboratory Animals as adopted and promulgated by the National Institutes of Health and were approved by the local animal care committee (Karlsruhe, Germany).

\section{Experimental Design}

In a first experiment, we examined the effects of the $\mathrm{CB} 1$ receptor antagonist/inverse agonist SR141716 (SR; Rimonabant) and the synthetic cannabinoid receptor agonist WIN 55 212-2 (WIN) on the well-established PAS procedure in rats. Therefore, rats received a single daily injection of 
$1 \mathrm{mg} / \mathrm{kg} \mathrm{SR} /$ vehicle or $0.3 \mathrm{mg} / \mathrm{kg}$ WIN/vehicle during training (SR experiment: SR $n=16$, vehicle $n=12$; WIN experiment: WIN $n=9$, vehicle $n=12$ ). As it is well known that the first injection of cannabinoid agonists in drug-naive rodents may be perceived as aversive (Schneider and Koch, 2002; Chaperon and Thiebot, 1999), animals in the WIN experiment were additionally subjected to a single priming injection of $0.3 \mathrm{mg} / \mathrm{kg}$ WIN, $48 \mathrm{~h}$ before the initiation of the PAS training procedure. To exclude that chronic SR/WIN administration may affect the ASR amplitude per se, we additionally assessed the ASR in another cohort of animals that did not undergo reward-association training. These animals were also tested two times for their ASR: once before and once after chronic drug administration of WIN/ SR or the respective vehicle (WIN experiment: WIN $n=6$, vehicle: $n=9$; SR experiment: SR $n=6$, vehicle: $n=7$ ).

The following experiments were conducted in CB1 KO mice and their respective WT littermates. A first cohort of WT/KO mice was used for behavioral testing. Group I was tested for sweetened condensed milk (SCM) intake, PAS, and in an odor preference task (WT and CB1 KO: $n=11$ ). Animals were left undisturbed for 3-4 days between the behavioral tests. Group II was sham-trained and served as control during PAS testing (WT and CB1 KO: $n=7$ ). A second cohort of mice was used for the FosB/ $\Delta$ FosB experiment (WT: trained and sham-trained: $n=5$; $\mathrm{CB} 1$ KO: trained $n=4$, sham-trained $n=5$ ).

A bottle of SCM (Nestle AG, Frankfurt, Germany; diluted 1:3 with tap water) was used for all behavioral experiments as food reward. All animals were habituated to SCM in their home cage for $24 \mathrm{~h}$ at least 4 days before the start of behavioral testing or training, to avoid novelty-induced disliking of the reward.

\section{Drugs}

SR (generously provided by NIMH) was dissolved in ethanol and Tween-80, and then diluted with saline (1:1:18). WIN (Sigma-Aldrich, Taufkirchen, Germany) was dissolved in $0.1 \%$ Tween-80 and diluted in saline $(0.9 \%)$. Both drugs were administered intraperitoneally at a dose of $1 \mathrm{mg} / \mathrm{kg}$ (SR) and $0.3 \mathrm{mg} / \mathrm{kg}$ (WIN). SR was injected $30 \mathrm{~min}$, and WIN directly before training with an injection volume of $1 \mathrm{ml} / \mathrm{kg}$.

\section{Behavioral Testing}

PAS in rats. PAS testing occurred in a startle chamber (SR-LAB; San Diego Instruments, San Diego, CA) as described previously (Schneider and Spanagel, 2008; Schneider et al, 2010; Brand et al, 2012). For startle testing a white noise pulse was used as the startle stimulus $(40 \mathrm{~ms}, 100 \mathrm{~dB}$ sound pressure level (SPL)). An acclimatization time of $5 \mathrm{~min}$, during which the rats received no stimulus except for the background noise $(60 \mathrm{~dB})$, was followed by the presentation of five initial startle stimuli. The following test protocol consisted of 30 startle pulses with an intertrial interval randomized between 10 and $20 \mathrm{~s}$. Startle testing occurred two times in the presence of an odor cue (orange, essential oils; Primavera Life, Sulzberg, Germany), once before (= ASR baseline; ASR1) and again $48 \mathrm{~h}$ after odor-reward-association training (ASR2). The odor $(30 \mu \mathrm{l})$ was provided in a
Petri dish that was placed in the startle box during habituation. PAS was calculated as mean percent decrease over baseline ASR amplitude $(100-(100 \times$ mean ASR2 amplitude/mean ASR1 (baseline) amplitude)).

Reward-association training lasted 5 days. During each daily 90 min training session, rats were placed in single cages (Eurostandard type III) and experienced three odorreward presentations at random time points. The odor (orange, $15 \mu \mathrm{l}$ ) was supplied in a small Petri dish that was placed in the middle of the wire lid, $2 \mathrm{~cm}$ beneath the aperture of the SCM drinking bottle. After free access to the reward for $5 \mathrm{~min}$, the odor and the bottle were removed. Thirty minutes (SR experiment) or directly (WIN experiment) before the initiation of each training session, all animals received a single injection of the respective drug or vehicle.

PAS in CB1 KO and WT mice. Startle testing in mice did not differ from the protocol described above for rats, with the exception of the startle intensity ( $115 \mathrm{~dB}$ SPL). Rewardassociation training in mice lasted 5 days and differed slightly from our protocol for rats. Training was performed in the home cages of the mice. In a daily time frame of $6 \mathrm{~h}$ (from 1000 to 1600 hours), all mice experienced five odor-reward presentations at random time points. The odor (orange, $15 \mu \mathrm{l}$ ) was supplied in a small Petri dish placed in the middle of the wire lid, $2 \mathrm{~cm}$ beneath the aperture of the bottle. After free access to the reward for $5 \mathrm{~min}$ the odor and the bottle were removed.

To validate the novel PAS protocol in mice one additional group was tested under sham conditions. These animals underwent a sham-training procedure where they received over 5 days daily access to SCM for $25 \mathrm{~min}$ at random time points without simultaneous odor presentation. Exposure to the odor took place in a non-associative manner at least $4 \mathrm{~h}$ after/before SCM access.

Limited access SCM intake in CB1 KO and WT mice. Animals were tested for SCM intake in their home cage. On the test day, the body weight (BW) was measured and animals were placed back in their home cage. Here they received free access to a bottle of SCM for $30 \mathrm{~min}$. SCM intake was then calculated as $\mathrm{g}$ intake per $\mathrm{kg} \mathrm{BW}$.

Odor preference test in CB1 KO and WT mice. An odor preference task in an open field $\left(20 \times 20 \times 25 \mathrm{~cm}^{3}\right)$ was used to assess the ability for odor detection in CB1 KO mice. During testing two identically shaped glass salt shakers (with perforated metal lids), containing a filter paper soaked with $15 \mu \mathrm{l}$ water or lemongrass oil, were placed into the arena. Mice were habituated $24 \mathrm{~h}$ before testing to the arena and the (unscented) objects for $10 \mathrm{~min}$. For object preference testing, mice were allowed to explore the scented objects freely for $6 \mathrm{~min}$. Animals were videotaped and the exploration time (s) of each object was analyzed offline by an experimenter blind to the genotype of the mice. For the calculation of percentage odor discrimination, the exploration time of lemongrass-scented object was expressed as percentage of the total exploration time of both objects. 


\section{Immunohistochemistry}

FosB/AFosB stimulation and brain preparation. To measure FosB $/ \Delta$ FosB protein expression, mice were trained (or sham-trained, respectively) for 5 days to associate an orange odor with the presence of SCM as described before for the PAS training protocol. All animals were exposed $48 \mathrm{~h}$ after the last association training to the orange odor $(15 \mu \mathrm{l})$ for $10 \mathrm{~min}$. Animals were killed $90 \mathrm{~min}$ later by decapitation and brains were removed, quickly frozen in methylbutan for $90 \mathrm{~s}$, and stored at $-80^{\circ} \mathrm{C}$. Frozen brains were sliced in $12 \mu \mathrm{m}$ coronal sections on a Cryostat and immediately thaw-mounted onto Superfrost glass slides and stored at $-80^{\circ} \mathrm{C}$. Two reward-related brain regions, the nucleus accumbens (NAC) and the dorsal striatum (dStr), were chosen as regions of interest.

FosB/AFosB immunohistochemistry. Slides were defrosted and dried at room temperature before fixation in $4 \%$ PFA. Slides were washed in phosphate-buffered saline (PBS) and PBS with $0.1 \%$ Tween-20 (PBS-T). After 30 min incubation with $0.3 \% \mathrm{H}_{2} \mathrm{O}_{2}$ dissolved in methanol and subsequent washes in PBS/PBS-T, the primary monoclonal FosB antibody (no. 2251; New England Biolabs GmbH, Frankfurt, Germany) that recognizes both FosB and $\triangle$ FosB proteins was incubated at a concentration of $1: 400$ in PBS with $0.2 \%$ normal goat serum over night at $4{ }^{\circ} \mathrm{C}$. Slides were washed again in $\mathrm{PBS} / \mathrm{PBS}-\mathrm{T}$ and further processed with the rabbit IgG Vectastain ABC kit (Vector Laboratories, Burlingame, $\mathrm{CA}$ ) according to the manufacturer's manual. Slides were incubated with the secondary antibody for $1 \mathrm{~h}$ at room temperature, washed, and treated with avidin and biotin for $1 \mathrm{~h}$ at room temperature. Afterwards, sections were washed and immunohistochemically stained with diaminobenzidine (Sigma-Aldrich, St Louis, MO), and transferred through a graded series of ethanol solutions and xylol before being coversliped in Eukitt ( $\mathrm{O}$ Kindler $\mathrm{GmbH}$, Freiburg, Germany).

Images were captured using Axioskop 2 microscope (Zeiss, Jena, Germany) with a $\times 20$ air objective (NA 0.5) and an Olympus ColorView 3 camera. At a section plane level of +1.45 to $+1.35 \mathrm{~mm}$ from Bregma (Paxinos and Franklin, 2001), a $500 \times 500 \mu \mathrm{m}^{2}$ graticule was positioned at a discrete site within the dStr and $\mathrm{NAC}$ and all FosB/ $\Delta$ FosBlabeled cells in this selection were counted using ImageJ (Schneider et al, 2012). Owing to a high degree of functional heterogeneity in the dStr, a discrete site sampling was chosen over a randomized sampling in this study as this method has been used successfully in previous studies (eg, Arnold et al, 2001). The placement of the sampling areas is depicted in Figure 4. Counts from NAC and dStr were obtained from three random sections of each animal by manual counting through an unbiased observer. For each region the FosB/ $\Delta$ FosB expression level was standardized for the mean counting score of the sham-trained group (set to $100 \%$ ) and relative changes were calculated in \%. In WT mice $\mathrm{Fos} B / \Delta \mathrm{FosB}$ protein expression was assumed to be higher in the conditioned group compared with shamtrained animals (Friemel et al, 2010), whereas in CB1-KO mice no directed assumption could be made about the regulation of $\mathrm{Fos} B / \Delta \mathrm{FosB}$ protein. Therefore, data analysis was separately conducted for each genotype.

\section{Statistical Analysis}

Pharmacological effects of WIN and SR on percentage PAS in trained and untrained rats were analyzed by a two factorial analysis of variance (ANOVA) (drug $\times$ training), respectively. Genotype effects (WT/KO) on behavioral performance were evaluated using Student's $t$-tests, with exception for the time course of the ASR and PAS in KO and WT mice. These data as well as SCM intake during PAS training in the WIN and SR experiments were analyzed by repeated-measure ANOVA with the Student-Neuman-Keuls test as a post hoc analysis. The odor cue-induced stimulation of FosB $/ \Delta$ FosB expression in the NAC and $\mathrm{dStr}$ was analyzed for each genotype by Student's $t$-tests. According to our previous findings on c-Fos stimulation (Friemel et al, 2010), we expected to observe increased FosB/ $\Delta$ FosB expression after odor training in WT animals. Therefore, one-sided $t$-tests were applied for the data analysis in WT mice. All data are expressed as means \pm SEM. The level of statistical significance was defined as $p<0.05$. All statistical analyses were performed with IBM SPSS Statistics 20.

\section{RESULTS}

\section{Effects of SR141716 and WIN 55,212-2 on PAS and SCM Intake in Rats}

Statistical analysis indicated a significant difference in PAS between SR and trained and untrained, vehicle-treated rats (training effect: $\mathrm{F}_{1,37}=4.39, p=0.043$ ) (Figure 1a). Further post hoc comparisons revealed a significant higher PAS in trained, vehicle-treated rats compared with all other groups (compared with trained/SR: $p=0.038$, to untrained/vehicle: $p=0.021$, and to untrained/SR: $p=0.036$ ). Chronic SR treatment inhibited PAS, as PAS scores in these animals did not differ significantly from untrained controls (untrained/ vehicle: $p=0.48$; untrained/SR: $p=0.58$ ). In addition, both untrained groups did not differ in their percentage ASR reduction from baseline $(p=0.92)$, indicating no pharmacological effect of chronic SR injections on repeated ASR testing.

Similarly, chronic WIN treatment affected PAS only in the reward-association training conditions (Figure 1b). Statistical analysis indicated a significant training effect $\left(\mathrm{F}_{1,32}=24.65\right.$, $p=0.001)$. Further post hoc comparisons revealed a significant higher PAS in trained, WIN-treated rats compared with trained, vehicle-treated controls $(\mathrm{p}=0.008)$. Trained, vehicle-treated animals also showed higher PAS scores compared with untrained, vehicle-treated controls $(p=0.023)$. Chronic WIN treatment alone did not affect percentage reduction of baseline ASR in untrained rats, as these animals did not differ from untrained, vehicle-treated controls $(p=0.92)$. Startle values during testing were as follows (data are expressed as means \pm SEM): trained-SR: ASR $1=2789.7 \pm 396.9$, ASR $2=1740.5 \pm 422.4, \mathrm{VEH}:$ ASR $1=$ $2442.7 \pm 893.6, \quad$ ASR $2=1413.9 \pm 410.9 ; \quad$ WIN: $\quad$ ASR $1=$ $2439.8 \pm 333.1$, ASR $2=1215 \pm 247.9$, VEH: ASR $1=1951.6 \pm$ 374.9, ASR2 = 1501.7 \pm 328.4 ; untrained-SR: ASR $1=917.2 \pm$ 255.9, $\quad$ ASR $2=785.1 \pm 144.6, \quad$ VEH: $\quad$ ASR $1=793.6 \pm 91.9$, ASR2 $=744.7 \pm 115.8 ;$ WIN: ASR $1=681.5 \pm 118.9$, ASR2 $=$ $646.1 \pm 149.1$, VEH: ASR $1=660.1 \pm 89.3$, ASR2 $=655.1 \pm$ 113.7). 
a

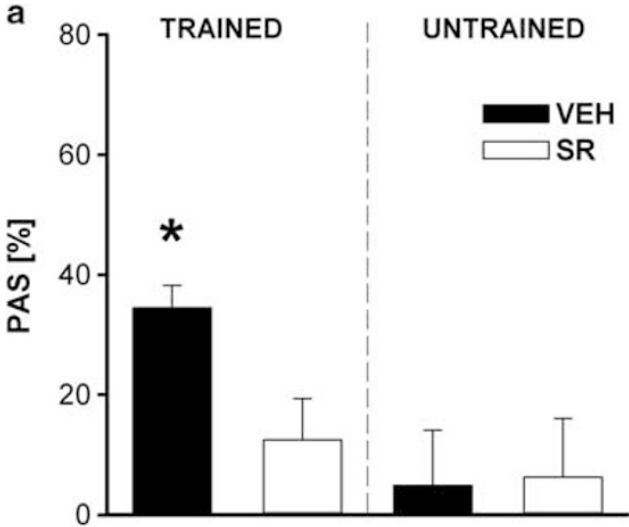

b

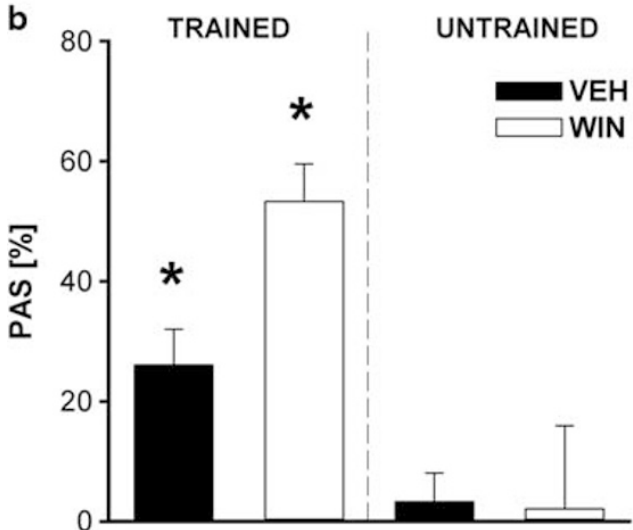

Figure I Effects of SRI4I7I6 (SR) and WIN 55212-2 (WIN) on pleasure attenuated startle (PAS) in rats. Percentage change from baseline acoustic startle response (ASR) by odor presentation was assessed in trained animals and untrained controls after chronic SR/vehicle (a) and WIN/vehicle (b) treatment. PAS was significantly affected in rats that received chronic SR $(I \mathrm{mg} / \mathrm{kg})$ treatment during appetitive odor conditioning compared with trained, vehicle-treated controls $(p=0.038)$. No significant treatment differences could be observed between SR and untrained, vehicle-treated animals $(p>0.05)$. Vehicle-treated animals that underwent reward-association training also differed significantly from their untrained, vehicle-treated controls $(p=0.02 \mathrm{I})$. Furthermore, chronic WIN $(0.3 \mathrm{mg} / \mathrm{kg})$ treatment during reward conditioning was found to increase PAS compared with vehicle-treated controls $(p=0.008)$. Chronic WIN treatment per se did not affect percentage ASR reduction in untrained animals ( $p>0.05)$, and in trained, vehicle-treated animals showed a significantly higher PAS than untrained, vehicle-treated controls $(p=0.023)$. Data are expressed as means \pm SEM ( $p<0.05$ is indicated by asterisks) (SR experiment-trained: $S R n=16$, vehicle $n=12$, untrained: $S R n=6$, vehicle $n=7$; WIN experiment: trained: $\mathrm{WIN} n=9$, vehicle $n=12$, untrained: WIN $n=6$, vehicle $n=9$ ).

SCM intake during PAS training was affected by SR with significantly lower intake rates throughout all five conditioning sessions compared with vehicle-treated rats $\left(\mathrm{F}_{1,26}=\right.$ 8.75, $p=0.007$; data not shown). However, no significant changes in SCM intake were observed between WIN-treated rats and their respective vehicle controls during PAS training $\left(\mathrm{F}_{1,16}=3.48, p=0.081\right.$; data not shown $)$.

\section{PAS and SCM Intake in CB1 KO and WT Mice}

PAS was significantly impaired in CB1 KO mice compared with WT animals $\left(t_{20}=-2.35, p=0.029\right)$ (Figure 2a). To validate the novel protocol for PAS in mice, a second group of animals was sham-trained and also tested two times for their ASR. We observed no significant differences between both genotypes in the sham training condition $\left(t_{12}=0.09\right.$, $p=0.93$ ) (Figure 2b), indicating that ASR amplitudes remain stable with reapeated testing to a similar extent in WT and CB1 KO mice. We also investigated the timeline of the startle sessions. The conditioned odor-cue reduced the ASR magnitude in WT animals during the second startle session (interaction effect: $\mathrm{F}_{2,20}=3.76, p=0.041$; post hoc analysis for startle trials: $0-10, p=0.007 ; 11-20, p<0.001$; $21-30, p=0.26)$, whereas no such difference was observed between the two startle sessions in CB1 KO mice $\left(\mathrm{F}_{2,20}=1.01, p=0.38\right)$ (Figure $2 \mathrm{c}$ ). No significant differences could be observed between the genotypes for baseline ASR amplitudes (values-CB1 KO: $89.6 \pm 11.1$; WT: $124.1 \pm 15.8$; $p=0.11)$. The time course of the PAS response differed significantly between the genotypes, with WT showing a lower percentage ASR inhibition during the entire test session (genotype effect: $\mathrm{F}_{1,40}=5.94, \mathrm{p}=0.024$; Figure $2 \mathrm{~d}$ ). Finally, free SCM intake was also significantly lower in CB1 KO mice compared with WT controls $\left(t_{20}=3.07, p=0.006\right.$; Figure 2e).

\section{Odor Discrimination in CB1 KO and WT Mice}

To assess the ability of CB1 KO mice to perceive odors, they were tested in an odor discrimination task. Both groups showed a preference for the scented object and no significant differences were observed between the genotypes in percentage odor discrimination $\left(t_{20}=-0.46, p=0.65\right)$ (Figure 3), indicating a similar response to odors in $\mathrm{CB} 1 \mathrm{KO}$ and WT mice.

\section{Odor-Induced FosB/DFosB Stimulation in $\mathrm{CB} 1 \mathrm{KO}$ and WT Mice}

Exemplary FosB/ $\Delta$ FosB stainings of the dStr from odortrained and sham-controlled animals of both genotypes are displayed in Figure 4. The induction of FosB/ $\Delta$ FosB expression by the odor cue was significantly higher in WT mice that underwent reward-association training compared with sham-trained mice in the NAC $\left(t_{8}=-1.99, p=0.04\right)$ (Figure 5a) and the $\mathrm{dStr}\left(t_{8}=-1.91, p=0.047\right)$ (Figure 5b). However, no such difference could be observed in CB1 KO mice (NAC: $t_{7}=0.88, p=0.41$; dStr: $t_{7}=-0.21, p=0.84$ ) (Figures $5 c$ and $d$ ), indicating that acute exposure to the reward-associated odor does not lead to a significant stimulation of reward-related brain regions in trained CB1 KO animals.

\section{DISCUSSION}

The present work demonstrates that the ECB system is an important modulator of hedonic reward processing, in addition to its well-known involvement in motivational and learning aspects of reward-related behaviors. Using a novel PAS paradigm in rats and mice, we here show that pharmacological inhibition or genetic invalidation of $\mathrm{CB} 1$ receptors decreases, whereas pharmacological activation of $\mathrm{CB} 1$ receptor enhances $\mathrm{PAS}$. Hence, $\mathrm{CB} 1$ receptor activity appears to be crucial for the induction of pleasant affective states that are able to attenuate an aversive reflex. 

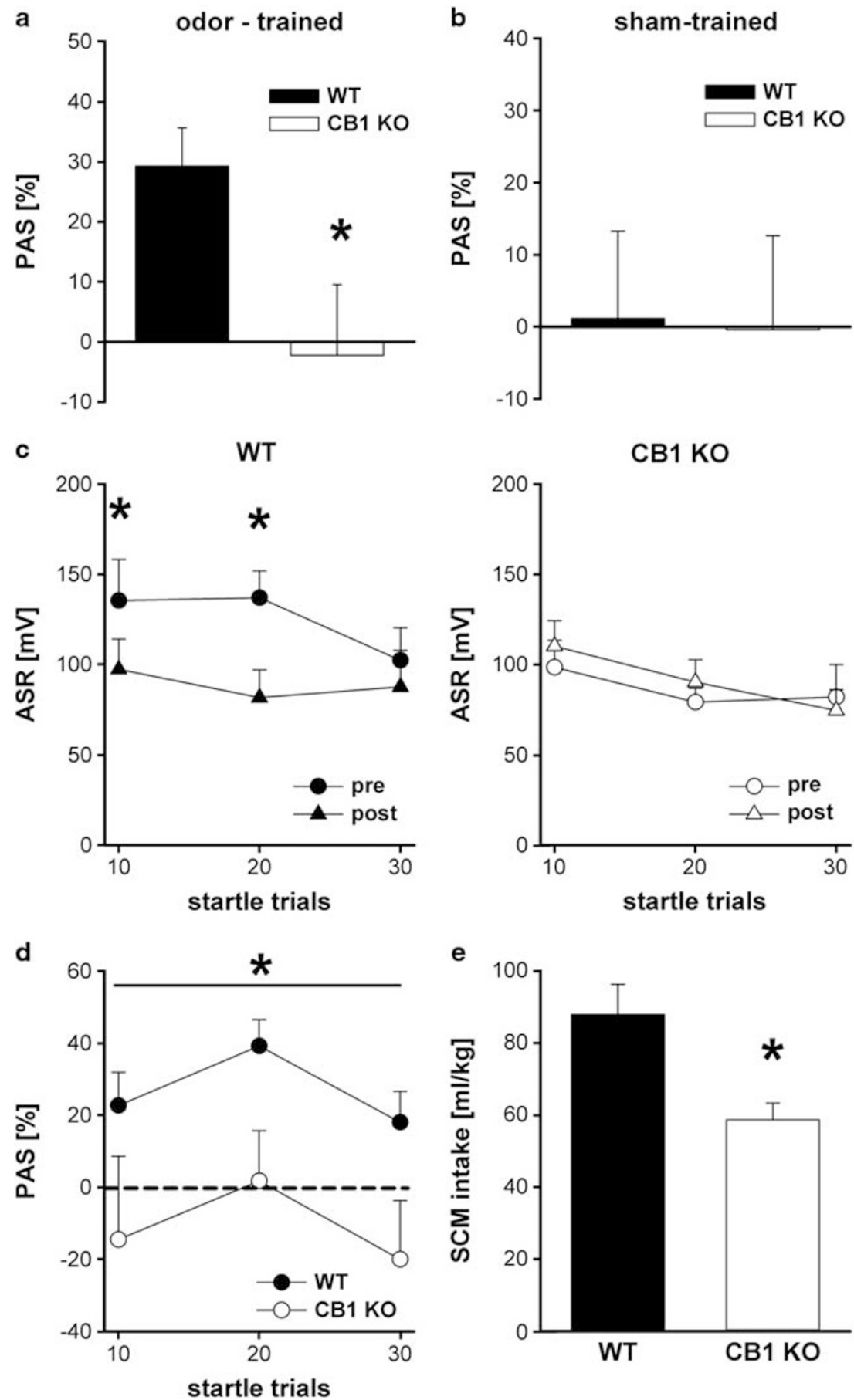

Figure 2 Pleasure attenuated startle (PAS), acoustic startle response (ASR), and sweetened condensed milk (SCM) intake in CBI knockout (KO) and wild-type (WT) mice. We observed a stable PAS response in WT mice; however, PAS was found to be almost absent in CBI KO mice (a). To control for potential variances in startle habituation between the test sessions, a second cohort of KO and WT mice underwent a sham training procedure without odor-reward association and were tested according the PAS protocol. No differences could be observed between the genotypes, indicating a similar ASR reponse after repeated testing in CBI KO and WT animals (b). The conditioned odor-cue attenuated the ASR magnitude in WT animals during the second startle session (c), whereas no such reduction could be observed in $\mathrm{CBI}$ KO mice (d). Accordingly, the time course in percentage PAS was significantly lower in $\mathrm{CBI} K \mathrm{KO}$ mice throughout the whole test session (e). Finally, free SCM intake was reduced in $\mathrm{CBI}$ KO mice compared with WT controls (f). Data are expressed as means \pm SEM ( $p<0.05$ is indicated by asterisks) (WT and CBI KO: $n=11$; sham training conditions-WT and CBI KO: $n=7$ ).

In a first experiment, we detected an inibitory effect of the $\mathrm{CB} 1$ receptor antagonist/inverse agonist SR in rats on the PAS response, when administered chronically during the PAS training procedure. SR-treated animals that were trained to associated the olfactory stimulus with the presence of the food reward did not differ in their percentage ASR reduction from untrained controls. In addition, intake of the palatable food reward (SCM) was also inhibited 
during reward-association training. The ability of SR to reduce feeding is well established and appears to be most pronounced for highly palatable food (Arnone et al, 1997; McLaughlin et al, 2005; Kirkham, 2009). However, the precise mechanisms through which $\mathrm{CB} 1$ receptor antagonism inhibits feeding has not been clarified so far. Specifically, it is not known whether reduced intake of palatable food is induced by decreased appetite and attenuated hedonic value of the food reward, or if side effects, such as incoordination, nausea, or substitute behaviors (ie, grooming, scratching, head twitching), which could interfere with food consumption and operant behavior, may play a role (Darmani and Pandya, 2000; Järbe et al, 2002; McLaughlin et al, 2005). A second experiment revealed a significantly enhanced PAS response after chronic treatment with the cannabinoid agonist WIN during reward-association training. Interestingly, despite its strong stimulatory effect on PAS, WIN did not increase the amount of SCM consumed during the training

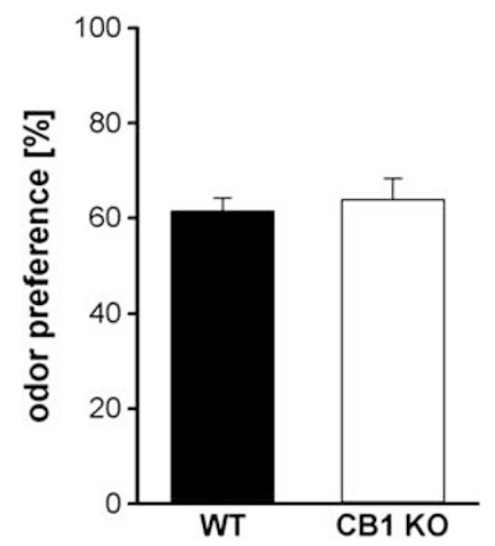

Figure 3 Odor preference in $\mathrm{CBI}$ knockout (KO) and wild-type (WT) mice. Both genotypes showed a similar preference for the scented object during odor discrimination testing, indicating a comparable ability for odor perception in $\mathrm{CBI} \mathrm{KO}$ and $\mathrm{WT}$ animals. Data are expressed as means \pm SEM (WT and CBI KO: $n=I I)$. procedure significantly. However, it may still have altered the hedonic value of the food reward and thereby increased the subjective value of the conditioned odor cue. Although it is well known that cannabinoid agonists generally enhance food intake, this effect has been reported to be biphasic and dose-dependent (Fattore et al, 2010). For the present study, a low dose of WIN was used $(0.3 \mathrm{mg} / \mathrm{kg})$; however, we cannot exclude that chronic administration of the drug may have induced side effects during SCM presentation that slightly interfered with the feeding rate (eg, locomotor slowing). The present findings indicate that evaluation of a food reward does not necessarily depend on consumption levels. Although WIN-treated rats consumed a similar amount of SCM than vehicle-treated controls, the subjective value assigned to the olfactory stimulus during SCM availability appeared to be more pronounced in the WIN group, as subsequent presentation of the odor induced a stronger inhibition of the aversive reflex in WIN-treated rats, compared with controls.

Our present findings of an attenuated PAS reponse in rats treated with SR during reward-association training, as well as an increased PAS after chronic WIN administration, indicate for the first time a direct involvement of $\mathrm{CB} 1$ receptor signaling specifically in the hedonic value of a food reward. Chronic treatment with either WIN or SR did not affect the ASR per se. Percentage reduction of baseline ASR after repeated testing did not differ compared with vehicletreated controls in animals that did not undergo rewardassociation training.

In addition to these pharmacological findings, we also show a more direct involvement of $\mathrm{CB} 1$ receptor signaling in hedonic processing by measuring PAS in CB1 KO mice. For this purpose, we introduced some methodological adaptations to our PAS protocol in rats, which enabled us to assess PAS in mice. We observed a strongly diminished PAS response in CB1 KO mice, even though startle habituation and odor detection did not differ between genotypes. Although baseline ASR amplitudes were slightly lower in

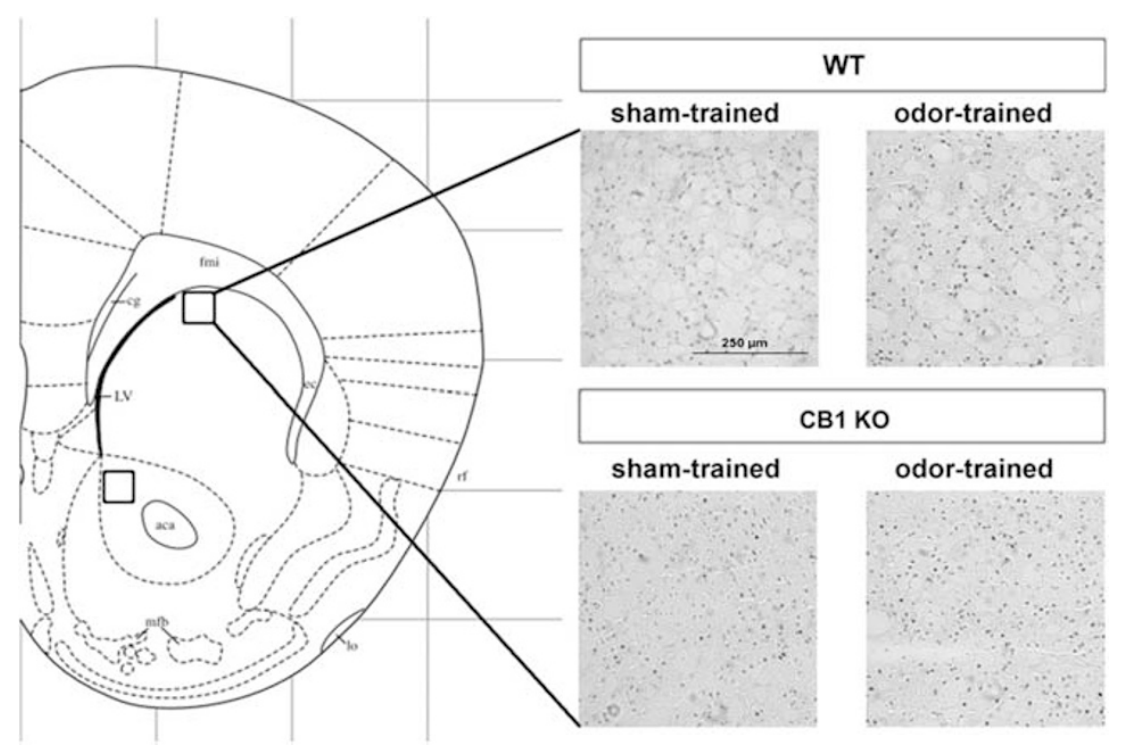

Figure 4 Striatal sampling sites and exemplary FosB/ $\Delta$ FosB staining. The schematic coronal brain section illustrates the steady position of sampling graticules $\left(500 \times 500 \mu \mathrm{m}^{2}\right)$ for the dorsal striatum (dStr) and nucleus accumbens (NAC) at a section plane corresponding to +1.45 to +1.35 from Bregma. Representative FosB/ $\Delta$ FosB stainings of the $\mathrm{dStr}$ from sham- and odor-trained mice of both genotypes are shown on the right side. 

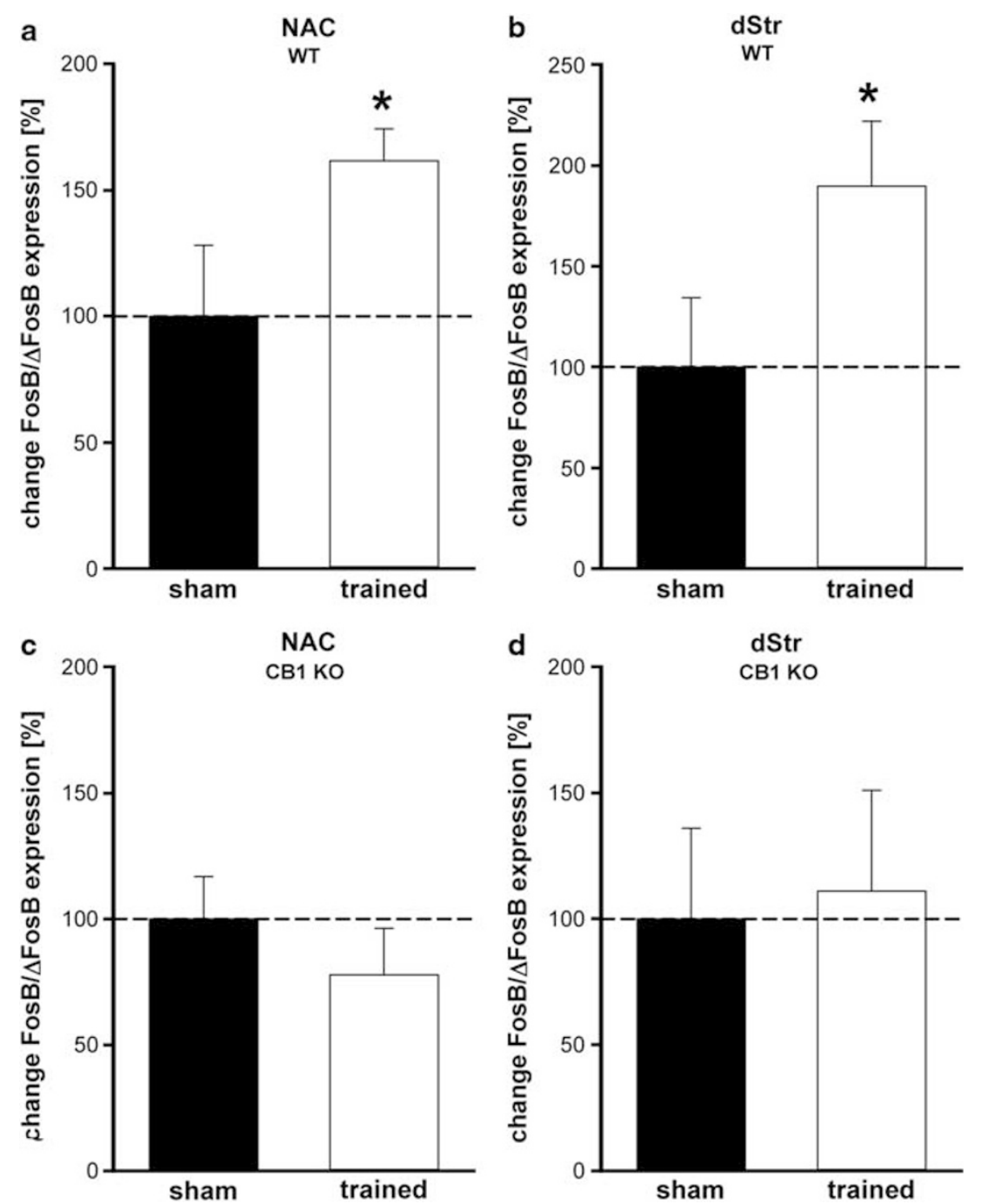

Figure 5 FosB/ $\Delta$ FosB stimulation by appetitive odor-cue presentation in CBI knockout (KO) and wild-type (WT) mice. The conditioned odor cue was found to significantly enhance FosB/ $\Delta$ FosB expression in trained WT animals in the nucleus accumbens (NAC) (a) and dorsal striatum (dStr) (b) compared to sham-trained controls. No such increased expression could be observed for CBI KO mice, neither in the NAC (c) nor the dStr (d). Data are expressed as means \pm SEM ( $p<0.05$ is indicated by asterisks) (WT: trained and sham-trained: $n=5 ; C B \mid K O$ : trained $n=4$, sham-trained $n=5)$.

KO mice compared with WT animals, this difference did not reach statistical significance. However, ASR amplitudes were still sufficiently high in CB1 KO animals to exclude potential floor effects. SCM intake was also significantly reduced in $\mathrm{CB} 1 \mathrm{KO}$ animals. Previous studies using CB1 KO animals indicated that lack of the $\mathrm{CB} 1$ receptor does not lead to marked changes in BW or food consumption in mice with ad libitum access to food (Ledent et al, 1999; Zimmer et al, 1999; Marsicano et al, 2002), although profound differences were observed in adipose mass between CB1 KO and WT mice (Cota et al, 2003). After food restriction, CB1 $\mathrm{KO}$ mice were found to eat less than their wild-type littermates (Di Marzo et al, 2001), have lower break points under PR schedules of sucrose delivery (Sanchis-Segura et al, 2004), and show decreased sucrose preference and consumption in a free-choice procedure (Sanchis-Segura et al, 2004; Poncelet et al, 2003). Interestingly, these effects appear to be most pronounced for highly palatable foods and liquids, as operant behavior for water and lab chow is not altered between the genotypes (Soria et al, 2005; Holter et al, 2005).
The use of the PAS paradigm in CB1 KO mice now allows a direct assessment of the impact of $\mathrm{CB} 1$ receptor signaling on the hedonic value of a food reward. This procedure excludes potential confounding factors, such as motoric requirements, motivational drive during operant responding, and acute sensory perception, as testing occurs in the absence of the reward and results in an attenuation of a physiological reflex (Koch et al, 1996; Schneider and Spanagel, 2008). The present data further indicate that ECB signaling is not only interfering with appetite and motivation, but rather modulates the hedonic value of palatable food. This is in line with different taste reactivity studies reporting that an inhibition of CB1 receptors decreases, whereas cannabinoid agonists increases, consumption and oral 'liking' responses for palatable liquids (Jarrett et al, 2006; Mahler et al, 2007). The importance of CB1 receptor availability for the mediation of PAS is also supported by an earlier more indirect observation of rat strain differences in PAS performance, which coincided with differences in $\mathrm{CB} 1$ receptor expression and cannabinoid-stimulated 
extracellular-regulated kinase phosphorylation (Brand et al, 2012).

In a final experiment, we examined the ability of the appetitive odor-cue to induce the immediate-early gene Fos $B / \triangle F o s B$ in reward-related brain sites. Acute exposure to natural rewards and drugs of abuse rapidly induces all Fos family members in the NAC and dStr, including FosB (Chao and Nestler, 2004). In an earlier study, we observed increased c-Fos expression in these regions after acute presentation of an appetitively conditioned odor cue in rats (Friemel et al, 2010). With the antibody used in the present study, we were not able to distinguish between FosB and $\Delta$ FosB. However, as exposure to the conditioned odor occurs only once for $10 \mathrm{~min}$, and $\Delta \mathrm{FosB}$ is well known to accumulate with time, particularly after chronic drug/ reward exposure (Chao and Nestler, 2004), we assume that our findings mainly represent expression of FosB, although this needs to be clarified in future studies. A recent study demonstrated that presentation of spatial cues associated with cocaine reward increased FosB expression in the NAC (El Rawas et al, 2012), with higher expression rates reflecting enhanced preference for the drug paired compartment. Our present data show a similar rise in $\mathrm{Fos} B / \Delta \mathrm{FosB}$ expression in the NAC and dStr in WT mice after presentation of a conditioned reward cue. However, the conditioned odor did not stimulate FosB/ $\Delta$ FosB expression in CB1 KO animals compared with sham-trained controls, further supporting a crucial role of $\mathrm{CB} 1$ receptor signaling in the processing of reward cues in reward-related brain structures.

Not much is known on the neurobiology of PAS so far. Previous studies in rats indicated that 6-OHDA lesion of the NAC, but not excitotoxic lesion of the amygdala, prevent the attenuation of the ASR in the presence of a rewarding stimulus (Koch et al, 1996). However, blockade of NAC dopaminergic D1/D2 receptors after conditioning was found to have no effect on PAS, implying that dopamine is not necessary for the expression of this form of startle gating (Koch et al, 2000). We reported recently a strong inhibition of PAS after acute injection of the opioid receptor antagonist naloxone in rats (Schneider et al, 2010), indicating an important modulatory role of the endogenous opioid system in the mediation of PAS. It is therefore conceivable that ECB signaling may affect pleasure and appetitive emotions by an interactive cross-talk with the endogenous opioid system. Evidence indicates that cannabinoids and opioids in part use identical mechanisms to modulate various physiological processes, including nociception, reward processing, and appetite. This is also supported by the observation that the distribution of CB1 receptors, opioid receptors, and their endogenous ligands is similar within brain areas of the reward circuitry (for a review see Manzanares et al, 1999; Cota et al, 2006; Herkenham et al, 1991), and CB1 and the $\mu$-opioid receptor are colocalized, for example, in the NAC and the dStr (for a review see Parolaro et al, 2010; Lopez-Moreno et al, 2010).

The experience of pleasurable and also aversive emotions is crucial for individual and gene survival. Life-supporting events need to be reinforced by incentives, whereas aversive events that might lead to pain or harm must be avoided. The experience of pleasure is therefore essential to a normal sense of well-being and health, and pathological dysfunctions in these processes (eg, anhedonia) have been linked to various neuropsychiatric disorders, such as addiction, depression, and schizophrenia. A detailed knowledge on neurocircuits and mechanisms involved in the mediation of pleasure are therefore crucial for a better understanding of malfunctions in hedonic processing (Berridge and Kringelbach, 2008; Esch and Stefano, 2004; Kringelbach and Berridge, 2009). The present work has added substantially to the understanding of the neurobiology of pleasure, implicating an important involvement of the ECB system, and in particular CB1 receptor signaling and availability, in the mediation of hedonic affect.

\section{FUNDING AND DISCLOSURE}

The authors declare no conflict of interest.

\section{ACKNOWLEDGEMENTS}

We thank Christian Gluch for excellent technical assistance. The present work was funded by grants from the Deutsche Deutsche Forschungsgemeinschaft (DFG) to MS (SCHN 958/3-1 and SCHN 958/4-1) and AZ (FOR926).

\section{REFERENCES}

Ameri A (1999). The effects of cannabinoids on the brain. Prog Neurobiol 58: 315-348.

Arnold JC, Topple AN, Mallet PE, Hunt GE, McGregor IS (2001). The distribution of cannabinoid-induced Fos expression in rat brain: differences between the Lewis and Wistar strain. Brain Res 921: 240-255.

Arnone M, Maruani J, Chaperon F, Thiebot M, Poncelet M, Soubrie $P$ et al (1997). Selective inhibition of sucrose and ethanol intake by SR 1411716, an antagonist of central cannabinoid (CB1) receptors. Psychopharmacology 132: 104-106.

Barbano MF, Cador M (2007). Opioids for hedonic experience and dopamine to get ready for it. Psychopharmacology 191: 497-506.

Berridge KC, Kringelbach ML (2008). Affective neuroscience of pleasure: reward in humans and animals. Psychopharmacology 199: 457-480.

Brand T, Spanagel R, Schneider M (2012). Decreased reward sensitivity in rats from the Fischer344 strain compared to Wistar rats is paralleled by differences in endocannabinoid signaling. PLoS One 7: e31169.

Chao J, Nestler EJ (2004). Molecular neurobiology of drug addiction. Annu Rev Med 55: 113-132.

Chaperon F, Thiebot M (1999). Behavioral effects of cannabinoid agents in animals. Crit Rev Neurobiol 13: 243-281.

Cota D, Marsicano G, Tschop M, Grubler Y, Flachskamm C, Schubert $\mathrm{M}$ et al (2003). The endogenous cannabinoid system affects energy balance via central orexigenic drive and peripheral lipogenesis. J Clin Invest 112: 423-431.

Cota D, Tschop MH, Horvath TL, Levine AS (2006). Cannabinoids, opioids and eating behavior: the molecular face of hedonism? Brain Res Rev 51: 85-107.

Darmani NA, Pandya DK (2000). Involvement of other neurotransmitters in behaviors induced by the cannabinoid $\mathrm{CB} 1$ receptor antagonist SR 141716A in naive mice. J Neural Transm 107: 931-945.

Davis M (1998). Anatomic and physiologic substrates of emotion in an animal model. J Clin Neurophysiol 15: 378-387.

Davis M, Walker DL, Myers KM (2003). Role of the amygdala in fear extinction measured with potentiated startle. Ann NY Acad Sci 985: 218-232. 
Di Marzo V, Goparaju SK, Wang L, Liu J, Batkai S, Jarai Z et al (2001). Leptin-regulated endocannabinoids are involved in maintaining food intake. Nature 410: 822-825.

Egertova M, Cravatt BF, Elphick MR (2003). Comparative analysis of fatty acid amide hydrolase and $\mathrm{cb}(1)$ cannabinoid receptor expression in the mouse brain: evidence of a widespread role for fatty acid amide hydrolase in regulation of endocannabinoid signaling. Neuroscience 119: 481-496.

El Rawas R, Klement S, Salti A, Fritz M, Dechant G, Saria A et al (2012). Preventive role of social interaction for cocaine conditioned place preference: correlation with FosB/DeltaFosB and pCREB expression in rat mesocorticolimbic areas. Front Behav Neurosci 6: 8 .

Elphick MR (2012). The evolution and comparative neurobiology of endocannabinoid signalling. Philos Trans $R$ Soc Lond Ser B 367: 3201-3215.

Enkel T, Spanagel R, Vollmayr B, Schneider M (2010). Stress triggers anhedonia in rats bred for learned helplessness. Behav Brain Res 209: 183-186.

Esch T, Stefano GB (2004). The neurobiology of pleasure, reward processes, addiction and their health implications. Neuro Endocrinol Lett 25: 235-251.

Fattore L, Melis M, Fadda P, Pistis M, Fratta W (2010). The endocannabinoid system and nondrug rewarding behaviours. Exp Neurol 224: 23-36.

Friemel CM, Spanagel R, Schneider M (2010). Reward sensitivity for a palatable food reward peaks during pubertal development in rats. Front Behav Neurosci 4: 39.

Herkenham M, Lynn AB, de Costa BR, Richfield EK (1991). Neuronal localization of cannabinoid receptors in the basal ganglia of the rat. Brain Res 547: 267-274.

Herkenham M, Lynn AB, Little MD, Johnson MR, Melvin LS, de Costa BR et al (1990). Cannabinoid receptor localization in brain. Proc Natl Acad Sci USA 87: 1932-1936.

Holter SM, Kallnik M, Wurst W, Marsicano G, Lutz B, Wotjak CT (2005). Cannabinoid CB1 receptor is dispensable for memory extinction in an appetitively-motivated learning task. Eur J Pharmacol 510: 69-74.

Järbe TU, Andrzejewski ME, DiPatrizio NV (2002). Interactions between the CB1 receptor agonist Delta 9-THC and the CB1 receptor antagonist SR-141716 in rats: open-field revisited. Pharmacol Biochem Behav 73: 911-919.

Jarrett MM, Scantlebury J, Parker LA (2006). Effect of Delta(9)tetrahydrocannabinol on quinine palatability and AM251 on sucrose and quinine palatability using the taste reactivity test. Physiol Behav 90: 425-430.

Jarrett MM, Scantlebury J, Parker LA (2007). Effect of Delta(9)tetrahydrocannabinol on quinine palatability and AM251 on sucrose and quinine palatability using the taste reactivity test. Physiol Behav 90: 425-430.

Kirkham TC (2009). Cannabinoids and appetite: food craving and food pleasure. Int Rev Psychiatry 21: 163-171.

Koch M, Schmid A, Schnitzler HU (1996). Pleasure-attenuation of startle is disrupted by lesions of the nucleus accumbens. NeuroReport 7: 1442-1446.

Koch M, Schmid A, Schnitzler HU (2000). Role of the nucleus accumbens dopamine D1 and D2 receptors in instrumental and Pavlovian paradigms of conditioned reward. Psychopharmacology 152: 67-73.

Kringelbach ML, Berridge KC (2009). Towards a functional neuroanatomy of pleasure and happiness. Trends Cogn Sci 13: 479-487.

Lang PJ (1995). The emotion probe. Studies of motivation and attention. Am Psychol 50: 372-385.

Lang PJ, Bradley MM, Cuthbert BN (1990). Emotion, attention, and the startle reflex. Psychol Rev 97: 377-395.

Ledent C, Valverde O, Cossu G, Petitet F, Aubert JF, Beslot F et al (1999). Unresponsiveness to cannabinoids and reduced addic- tive effects of opiates in $\mathrm{CB} 1$ receptor knockout mice. Science (New York, NY) 283: 401-404.

Lopez-Moreno JA, Lopez-Jimenez A, Gorriti MA, de Fonseca FR (2010). Functional interactions between endogenous cannabinoid and opioid systems: focus on alcohol, genetics and drugaddicted behaviors. Curr Drug Targets 11: 406-428.

Mahler SV, Smith KS, Berridge KC (2007). Endocannabinoid hedonic hotspot for sensory pleasure: anandamide in nucleus accumbens shell enhances 'liking' of a sweet reward. Neuropsychopharmacology 32: 2267-2278.

Manzanares J, Corchero J, Romero J, Fernandez-Ruiz JJ, Ramos JA, Fuentes JA (1999). Pharmacological and biochemical interactions between opioids and cannabinoids. Trends Pharmacol Sci 20: 287-294.

Marsicano G, Wotjak CT, Azad SC, Bisogno T, Rammes G, Cascio MG et al (2002). The endogenous cannabinoid system controls extinction of aversive memories. Nature 418: 530-534.

McLaughlin PJ, Winston KM, Limebeer CL, Parker LA, Makriyannis A, Salamone JD (2005). The cannabinoid CB1 antagonist AM 251 produces food avoidance and behaviors associated with nausea but does not impair feeding efficiency in rats. Psychopharmacology (Berl) 180: 286-293.

Norrholm SD, Jovanovic T, Vervliet B, Myers KM, Davis M, Rothbaum BO et al (2006). Conditioned fear extinction and reinstatement in a human fear-potentiated startle paradigm. Learn Mem 13: 681-685.

Parolaro D, Rubino T, Vigano D, Massi P, Guidali C, Realini N (2010). Cellular mechanisms underlying the interaction between cannabinoid and opioid system. Curr Drug Targets 11: 393-405.

Paxinos G, Franklin KBJ (2001). The Mouse Brain in Stereotaxic Coordinates. 2nd edn Academic Press: San Diego, CA.

Poncelet M, Maruani J, Calassi R, Soubrie P (2003). Overeating, alcohol and sucrose consumption decrease in $\mathrm{CB} 1$ receptor deleted mice. Neurosci Lett 343: 216-218.

Salamone JD, Correa M, Farrar A, Mingote SM (2007). Effortrelated functions of nucleus accumbens dopamine and associated forebrain circuits. Psychopharmacology 191: 461-482.

Sanchis-Segura C, Cline BH, Marsicano G, Lutz B, Spanagel R (2004). Reduced sensitivity to reward in CB1 knockout mice. Psychopharmacology (Berl) 176: 223-232.

Schmid A, Koch M, Schnitzler HU (1995). Conditioned pleasure attenuates the startle response in rats. Neurobiol Learn Mem 64: $1-3$.

Schneider CA, Rasband WS, Eliceiri KW (2012). NIH Image to ImageJ: 25 years of image analysis. Nat Methods 9: 671-675.

Schneider M, Heise V, Spanagel R (2010). Differential involvement of the opioid receptor antagonist naloxone in motivational and hedonic aspects of reward. Behav Brain Res 208: 466-472.

Schneider M, Koch M (2002). The cannabinoid agonist WIN $55,212-2$ reduces sensorimotor gating and recognition memory in rats. Behav Pharmacol 13: 29-37.

Schneider M, Spanagel R (2008). Appetitive odor-cue conditioning attenuates the acoustic startle response in rats. Behav Brain Res 189: $226-230$.

Schultz W (2010). Dopamine signals for reward value and risk: basic and recent data. Behav Brain Funct 6: 24.

Solinas M, Goldberg SR, Piomelli D (2008). The endocannabinoid system in brain reward processes. Br J Pharmacol 154: 369-383. Soria G, Mendizabal V, Tourino C, Robledo P, Ledent C, Parmentier M et al (2005). Lack of CB1 cannabinoid receptor impairs cocaine self-administration. Neuropsychopharmacology 30: $1670-1680$.

Vrana SR, Spence EL, Lang PJ (1988). The startle probe response: a new measure of emotion? J Abnorm Psychol 97: 487-491.

Zimmer A, Zimmer AM, Hohman AG, Herkenham M, Bonner TI (1999). Increased mortality, hypoactivity, and hypoalgesia in cannabinoid CB1 receptor knockout mice. Proc Natl Acad Sci USA 96: 5780-5785. 